\title{
SYNTHESIS OF NOVEL ANTI-BACTERIAL 2,1-BENZOTHIAZINE 2,2-DIOXIDES DERIVED FROM METHYL ANTHRANILATE
}

\author{
MUHAMMAD SHAFIQ ${ }^{l}$, MUHAMMAD ZIA-UR-REHMAN ${ }^{2 *}$, ISLAM ULLAH KHAN ${ }^{1}$, \\ MUHAMMAD NADEEM ARSHAD ${ }^{l}$, SIRAJ AHMED KHAN
}

\author{
${ }^{1}$ Materials Chemistry Laboratory, Department of Chemistry, Government College University; Lahore-54000, Pakistan \\ ${ }^{2}$ Applied Chemistry Research Centre, PCSIR Laboratories Complex, Lahore-54600, Pakistan \\ ${ }^{3}$ Microbiology Laboratories, Qarshi Research International, Hattar, Pakistan
}

(Received: October 14, 2010 - Accepted: January 26, 2011)

\begin{abstract}
A convenient ultrasound assisted synthesis of new anti-bacterial $N$-benzylidene- $N$ '-(1-methyl-2, 2-dioxo-2,3-dihydro- $1 H$-2 $2 \lambda^{6}$-benzo[c] [1,2]thiazin-4-ylidene)hydrazines from methyl anthranilate is reported. Methyl anthranilate was reacted with methane sulfonyl chloride, followed by $N$-alkylation and subsequent base catalyzed cyclization. Hydrazinolysis of the cyclized products followed by their ultrasound mediated condensations with different benzaldehydes yielded the title compounds. Some of these compounds showed good anti-bacterial activity against Pseudomonas aeruginosa, Salmonella typhimurium and Staphylococcus aureus.
\end{abstract}

Key Words: 2,1-Benzothiazine 2,2-dioxides, Schiff bases, Anti-bacterial activity, Methyl anthranilate.

\section{INTRODUCTION}

Sulfonamides are well known for their enormous potential as pharmaceutical and agricultural agents due to their versatile nature of biological activities $^{1}$ while cyclic sulfonamides are important as chiral auxiliaries ${ }^{2,3}$ and therapeutic compounds ${ }^{4,5}$. Among these, benzothiazines, due to their diverse biological activities are gaining much attention since the very first synthesis by Braun et $a l^{6}$. Many of the benzothiazine based drugs e.g., Piroxicam ${ }^{\otimes}$, Droxicam $^{\circledR}$ and Lornoxicam ${ }^{\circledR}$ (Fig 1) are now-a-days in the market due to their effective role as analgesic and anti-inflammatory agents.
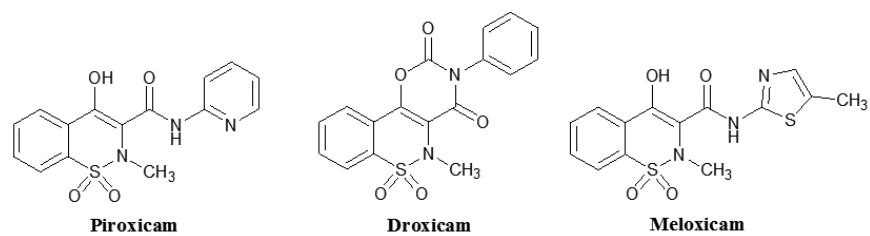

Figure 1: Examples of some benzothiazine based drugs

2,1-Benzothiazine 2,2-dioxides are now becoming important and are being reported in literature due to their applications in the synthesis of medicinally important natural products and new chiral ligands for catalysis and molecular recognition?. Among these, 3,4-dihydro-2,1-benzothiazine 2,2-dioxide derivatives have been reported to possess potent biological activities such as lipoxygenase inhibition and are being used as drugs for treating heart diseases ${ }^{8}$ (Fig. 2).<smiles>[R]c1ccc2c(c1[R])CC[S+]([O])(=O)N2[R]</smiles>

$$
\begin{aligned}
& \mathrm{R}^{1}=\mathrm{H}, \text { Benzyl } \\
& \mathrm{R}^{2}=\mathrm{H}, \text { Alkoxy, Hydroxy } \\
& \mathrm{R}^{3}=\mathrm{H}, \text { Piperazinyl }
\end{aligned}
$$

Figure 2: 3,4-Dihydro-2,1-benzothiazine 2,2-dioxide derivatives

Carbohydrazides are becoming popular day by day in the field of drug development due to their easy cyclization and condensations 9 . Up till now, a large number of such compounds have been synthesized with significant antimicrobial activities ${ }^{10,11}$. In continuation of our on-going research on various biologically active benzothiazine derivatives $\mathrm{s}^{12-14}$, synthesis of $\mathrm{N}$-benzylidene$N^{\prime}$-(1-methyl-2,2-dioxo-2,3-dihydro- $1 H$ - $2 \lambda^{6}$-benzo[c][1,2] thiazin-4-ylidene)hydrazines has been carried out to get a series of possible biologically active compounds. Besides, characterization of the newly synthesized compounds through spectroscopic techniques and single crystal X-ray analysis, these were also subjected to the preliminary evaluation for their anti-bacterial potential due to their carbohydrazide functionalities ${ }^{10,11}$.

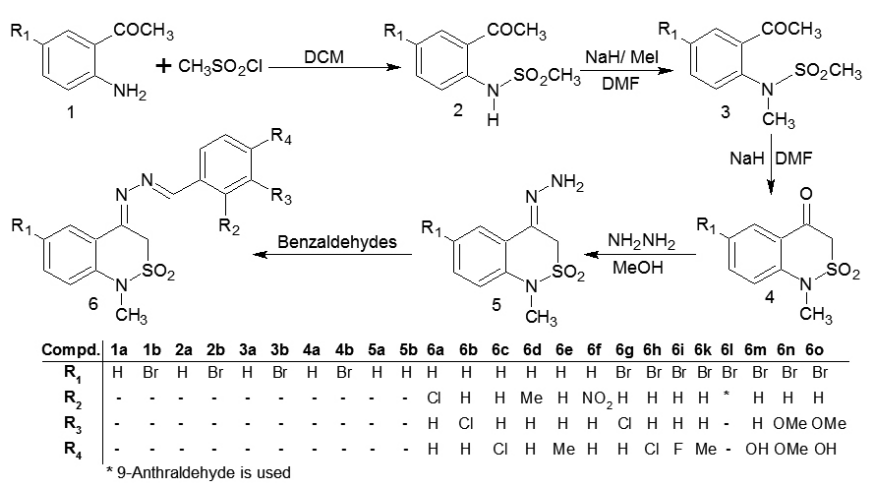

Figure 3: Synthesis of $N$-benzylidene- $N$ '-(1-methyl-2, 2-dioxo-2,3dihydro- $1 H-2 \lambda^{6-}$ benzo[c] $][1,2]$ thiazin-4-ylidene)-hydrazines 


\section{EXPERIMENTAL}

All the chemicals were purchased from E. Merck, BDH or Fluka and used without purification. However, solvents were purified through distillation. ${ }^{1} \mathrm{H}$ NMR spectra were recorded on a Bruker DPX-400 instrument at $400 \mathrm{MHz}$. Chemical shifts are reported in ppm referenced to the residual solvent signal. Melting points were recorded on an electrothermal (Griffin 1090) apparatus and are reported as uncorrected. IR spectra were recorded on a Perkin Elmer 1600-FT spectrometer. Mass spectra were taken on a Jeol SX-102 instrument. Ultrasonic mediated reactions were carried out in Clifton Ultrasonic Bath $(2 \mathrm{x}$ T2A, 300W, DU-4) made by Nickel Electro Ltd, Weston-S-Mare Somerset, England.

\section{$N$-(2-Acetylphenyl)methanesulfonamide (2a):}

A mixture of methanesulfonyl chloride $(0.55 \mathrm{~g} ; 4.8 \mathrm{mmoles})$, methyl anthranilate $(0.72 \mathrm{~g} ; 4.8 \mathrm{mmoles})$ and dichloromethane $(30 \mathrm{ml})$ was allowed to react in an ultrasonic bath at $40^{\circ} \mathrm{C}$ for a period of 30 minutes till the completion of reaction (as indicated by TLC). The contents were washed twice with water and dried. Evaporation of the solvent under vacuum followed by recrystallization of the crude product from ethanol afforded white crystalline solid. Yield: $90 \%$. M.p. $88-89^{\circ} \mathrm{C}$ (Lit M.p. $\left.89-90^{\circ} \mathrm{C}\right)^{15}$; IR $(\mathrm{KBr}) \mathrm{cm}^{-1}: 3248$, $3049,1740,1342,1244$.

\section{$N$-(2-Acetyl-4-bromophenyl)methanesulfonamide (2b):}

A mixture of methanesulfonyl chloride $(0.55 \mathrm{~g} ; 4.8 \mathrm{mmol}), 1$-(2-amino5-bromophenyl)ethanone (1.10 g; $4.8 \mathrm{mmoles})$ and dichloromethane $(10 \mathrm{ml})$ was allowed to react in an ultrasonic bath at $40^{\circ} \mathrm{C}$ for a period of 3.5 hours till the completion of reaction (as indicated by TLC). The contents were washed twice with water and dried. Evaporation of the solvent under vacuum and recrystallization of the crude product from ethanol afforded white crystalline solid. Yield: $82 \%$. M.p. $92-93^{\circ} \mathrm{C}$. ${ }^{1} \mathrm{H}$ NMR (MeOD) $\delta: 2.70$ (s, 3H, $\mathrm{SCH}_{3}$ ), 3.50 $\left(\mathrm{s}, 3 \mathrm{H}, \mathrm{OCH}_{3}\right), 5.21(\mathrm{~s}, 1 \mathrm{H}, \mathrm{NH}), 8.10(\mathrm{~s}, 1 \mathrm{H}, \mathrm{ArH}), 7.52-7.70(\mathrm{dd}, 2 \mathrm{H}, \mathrm{Ar} H)$; IR ( $\mathrm{KBr}) \mathrm{cm}^{-1}: 3250,3050,2950,1740,1345$. MS m/z: 307( $\left.\mathrm{M}^{+}\right), 309\left(\mathrm{M}^{+}+2\right)$.

\section{$\mathrm{N}$-(2-Acetylphenyl)- $\mathrm{N}$-methylmethanesulfonamide (3a):}

A mixture of $N$-(acetylphenyl)methanesulfonamide (2a) (7.56 g; 33.0 mmoles), dimethylformamide $(25.0 \mathrm{ml})$ and sodium hydride $(1.58 \mathrm{~g} ; 66.0$ mmoles) was stirred for 45 minutes followed by the addition of methyl iodide $(9.37 \mathrm{~g} ; 66.0$ mmoles). The contents were stirred at room temperature for a further period of 85 minutes and were poured into ice cold hydrochloric acid $(3 \mathrm{~N})$ which was extracted with dichloromethane. Solvent was evaporated under reduced pressure to get the product. Yield: $62.0 \%$; M.p. $55^{\circ} \mathrm{C}$ (Lit. M.p. $\left.55-56^{\circ} \mathrm{C}\right)^{16}$; IR (KBr) cm ${ }^{-1}: 3246,3044,1706,1342$.

$\mathrm{N}$-(2-Acetyl-4-bromophenyl)- $\mathrm{N}$-methylmethanesulfonamide (3b):

A mixture of $N$-(2-acetyl-4-bromophenyl)methanesulfonamide (2b) (10.16 g; 33.0 mmoles), dimethylformamide $(25.0 \mathrm{ml})$ and sodium hydride $(1.58 \mathrm{~g}$; 66.0 mmoles) was stirred for 45 minutes followed by the addition of methyl iodide $(9.37 \mathrm{~g}$; $66.0 \mathrm{mmoles})$. The contents were stirred at room temperature for a further period of 15 hours and were poured into ice cold hydrochloric acid $(3 \mathrm{~N})$ which was extracted with dichloromethane. Solvent was evaporated under reduced pressure to get the product. Yield: $67.0 \%$; M.p. $64-65^{\circ} \mathrm{C}$. (Lit. M.p. $\left.64^{\circ} \mathrm{C}\right)^{17}$; IR $(\mathrm{KBr}) \mathrm{cm}^{-1}: 3242,3043,1732,1337$. (4a):

1-Methyl-2,2-dioxo-2,3-dihydro-1 $H$-2 $\lambda^{6}$-benzo $[c][1,2]$ thiazin-4-one

To a suspension of $\mathrm{n}$-hexane washed sodium hydride $(2.30 \mathrm{~g} ; 96.0$ mmoles $)$ in dry DMF $(30 \mathrm{ml})$, a solution of $N$-(2-acetylphenyl)- $N$ methylmethanesulfonamide (3a) (11.66 g; 48.0 mmoles) in dry DMF (70 ml) was added and stirred at room temperature for 1.5 hours. After completion of the reaction (as indicated by TLC), the contents were poured into cold hydrochloric acid $(3 \mathrm{~N})$ to get the precipitates which were dried at room temperature to get the title compound. Yield: $91 \%$; M.p. 118-119 ${ }^{\circ} \mathrm{C}$ (Lit M.p. $\left.120^{\circ} \mathrm{C}\right)^{18}$; IR (KBr) cm ${ }^{-1}: 3250,3052,1734,1680,1342$

6-Bromo-1-methyl-2,2-dioxo-2,3-dihydro- $1 H-2 \lambda^{6}$-benzo $[c][1,2]$ thiazin-4-one (4b):

To a suspension of $\mathrm{n}$-hexane washed sodium hydride $(2.30 \mathrm{~g} ; 96.0$ mmoles) in dry DMF $(30 \mathrm{ml})$, solution of $N$-(2-acetyl-4-bromophenyl)- $N$ methylmethanesulfonamide (3b) (13.92 g; 48.0 mmoles) in dry DMF (70 ml) was added and stirred at room temperature for 3.5 hours. After completion of the reaction (as indicated by TLC), contents were poured into cold hydrochloric acid $(3 \mathrm{~N})$ to get the precipitates which were dried at room temperature to get the title compound. Yield: $90 \%$; M.p. $108-109^{\circ} \mathrm{C}$ (Lit M.p. $110^{\circ} \mathrm{C}$ ) ${ }^{19}$; IR (KBr) $\mathrm{cm}^{-1}: 3254,3055,1739,1690,1342$.

(1-Methyl-2,2-dioxo-2,3-dihydro-1 $H$-2 $\lambda^{6}$-benzo $[c][1,2]$ thiazin-4ylidene)-hydrazine (5a):

A mixture of 1-methyl-2,2-dioxo-2,3-dihydro- $1 H$ - $2 \lambda^{6}$-benzo[c] $[1,2]$ thiazin-4-one (4a) (10.60 g; 50.0 mmoles), hydrazine hydrate $(85 \%)(5.0 \mathrm{ml})$ and ethanol $(200 \mathrm{ml})$ was allowed to react at $45^{\circ} \mathrm{C}$ in an ultrasound reaction bath for a period of 35 minutes. After completion of the reaction, excess hydrazine and solvent were removed under vacuum. The crude product obtained was washed with water and dried. Light brown solid; Yield: 88\%; M.p. $139-141^{\circ} \mathrm{C}$. ${ }^{1} \mathrm{H}$ NMR $\left(\mathrm{CDCl}_{3}\right) \delta: 3.37$ (s, 3H, N-CH $\left.)_{3}\right), 4.50\left(\mathrm{~s}, 2 \mathrm{H}, \mathrm{CH}_{2}\right), 5.21\left(\mathrm{~s}, 2 \mathrm{H}, \mathrm{NH}_{2}\right)$, 6.88-7.92 (m, 4H, $\operatorname{Ar} H$ ). IR (KBr): 3410, 1680, 1384, $1160 \mathrm{~cm}^{-1}$; MS m/z: $225.03\left[\mathrm{M}^{+}\right]$; Anal. calcd for $\mathrm{C}_{9} \mathrm{H}_{11} \mathrm{~N}_{3} \mathrm{SO}_{2}$ : C, 48.00; H, 4.89; N, 18.67; Found: C, $48.01 ; \mathrm{H}, 4.90 ; \mathrm{N}, 18.68$.

(6-Bromo-1-methyl-2,2-dioxo-2,3-dihydro-1H-2 $\lambda^{6}$-benzo $[c][1,2]$ thiazin-4-ylidene)-hydrazine (5b):

Yellow needle like crystalline compound was prepared using procedure employed for 5a. Yield: 74\%; M.p. 178-179 ${ }^{\circ} \mathrm{C} ;{ }^{1} \mathrm{H} \mathrm{NMR}\left(\mathrm{CDCl}_{3}\right) \delta: 3.39(\mathrm{~s}$, $\left.3 \mathrm{H}, \mathrm{NCH}_{3}\right), 4.46\left(\mathrm{~s}, 2 \mathrm{H}, \mathrm{CH}_{2}\right), 5.15$ (s, 2H, $\left.\mathrm{NH}_{2}\right), 6.92-8.10$ (m, 3H, $\left.\mathrm{ArH}\right)$. IR (KBr): $3415,1685,1380,1155 \mathrm{~cm}^{-1}$; MS $m / z: 303\left[\mathrm{M}^{+}\right], 305\left[\mathrm{M}^{+}+2\right]$; Anal. calcd for $\mathrm{C}_{9} \mathrm{H}_{10} \mathrm{~N}_{3} \mathrm{SO}_{2} \mathrm{Br}$ : C, 35.53; H, 3.29; N, 13.82; Found: C, 35.50; H, $3.32 ; \mathrm{N}, 13.79$.

General procedure for the synthesis of $N$-benzylidene- $N$-(1-methyl2,2-dioxo-2,3-dihydro- $1 H$-2 $\lambda^{6}$-benzo[c][1,2] thiazin-4-ylidene)-hydrazines (6a-o)

(Using thermal conditions):

A mixture of (1-methyl-2,2-dioxo-2,3-dihydro-1H-2 $\lambda^{6}$-benzo[c] $[1,2]$ thiazin-4-ylidene)-hydrazine (5a) or (6-bromo-1-methyl-2,2-dioxo-2,3dihydro- $1 H$-2 $\lambda^{6}$-benzo $[c][1,2]$ thiazin-4-ylidene)-hydrazine (5b) $(2.0 \mathrm{mmol})$, corresponding aromatic aldehyde $(2.0 \mathrm{mmol})$, methanol $(50 \mathrm{ml})$ and glacial acetic acid ( 2 drops) was refluxed till completion of the reaction (for yields, reaction conditions $\&$ reaction times, see Table 1). The contents were cooled to $5^{\circ} \mathrm{C}$ in an ice bath, filtered and the solids were washed with cold methanol to get the pure compound.

\section{(Using ultrasonic waves)}

A mixture of (1-methyl-2,2-dioxo-2,3-dihydro- $1 H$-2 $2 \lambda^{6}$-benzo[c] $[1,2]$ thiazin-4-ylidene)-hydrazine (5a) or (6-bromo-1-methyl-2,2-dioxo-2,3dihydro- $1 H-2 \lambda^{6}$-benzo $[c][1,2]$ thiazin-4-ylidene)-hydrazine (5b) $(2.0 \mathrm{mmol})$, corresponding aromatic aldehydes $(2.0 \mathrm{mmol})$, methanol $(50 \mathrm{ml})$ and glacial acetic acid (2 drops) taken in a loosely screw capped test tube was immersed in an ultrasonic reaction bath (for yields, reaction conditions and reaction times, see Table 1). The contents were cooled and washed with cold methanol to get the pure compound.

$\mathrm{N}$-(2-Chloro-benzylidene)- $\mathrm{N}^{\prime}$-(1-methyl-2,2-dioxo-2,3-dihydro- $1 \mathrm{H}$ $2 \lambda^{6}$-benzo $[c][1,2]$ thiazin-4-ylidene)-hydrazine (6a):

Yellow crystals; M.p. $296-298^{\circ} \mathrm{C} .{ }^{1} \mathrm{H}$ NMR $\left(\mathrm{CDCl}_{3}\right) \delta: 3.42$ (s, $3 \mathrm{H}$, $\left.\mathrm{N}-\mathrm{CH}_{3}\right), 4.86\left(\mathrm{~s}, 2 \mathrm{H}, \mathrm{CH}_{2}\right), 7.12-8.20(\mathrm{~m}, 8 \mathrm{H}, \mathrm{Ar} H), 8.59$ (s, $\left.1 \mathrm{H}, \mathrm{NCH}\right)$. IR (KBr): $3508,1640,1419,1328,1148 \mathrm{~cm}^{-1}$; MS m/z: $347\left[\mathrm{M}^{+}\right], 349\left[\mathrm{M}^{+}+2\right]$; Anal. calcd for $\mathrm{C}_{16} \mathrm{H}_{14} \mathrm{~N}_{3} \mathrm{SO}_{2} \mathrm{Cl}$ : C, 55.25; H, 4.03; N, 12.09; Found: C, 55.26; $\mathrm{H}, 4.03 ; \mathrm{N}, 12.06$.

$\mathrm{N}$-(3-Chloro-benzylidene)- $\mathrm{N}$-(1-methyl-2,2-dioxo-2,3-dihydro- $1 \mathrm{H}$ $2 \lambda^{6}$-benzo[c] $[1,2]$ thiazin-4-ylidene)-hydrazine $(6 \mathrm{~b})$ :

Yellow powder; M.p. $198-200{ }^{\circ} \mathrm{C} .{ }^{1} \mathrm{H}$ NMR $\left(\mathrm{CDCl}_{3}\right) \delta: 3.38$ (s, $3 \mathrm{H}$, $\left.\mathrm{N}-\mathrm{CH}_{3}\right), 4.85\left(\mathrm{~s}, 2 \mathrm{H}, \mathrm{CH}_{2}\right), 7.12-7.70(\mathrm{~m}, 8 \mathrm{H}, \mathrm{ArH}), 8.56(\mathrm{~s}, 1 \mathrm{H}, \mathrm{NCH})$. IR (KBr): $3518,1632,1426,1312,1155 \mathrm{~cm}^{-1}$; MS m/z: $347\left[\mathrm{M}^{+}\right], 349\left[\mathrm{M}^{+}+2\right]$; Anal. calcd for $\mathrm{C}_{16} \mathrm{H}_{14} \mathrm{~N}_{3} \mathrm{SO}_{2} \mathrm{Cl}$ : C, 55.25; H, 4.03; N, 12.09; Found: C, 55.24; $\mathrm{H}, 4.05 ; \mathrm{N}, 12.10$.

$\mathrm{N}$-(4-Chloro-benzylidene)- $\mathrm{N}$ '-(1-methyl-2,2-dioxo-2,3-dihydro- $1 \mathrm{H}$ $2 \lambda^{6}$-benzo $[c][1,2]$ thiazin-4-ylidene)-hydrazine (6c):

Yellow crystals; M.p. $203-204^{\circ} \mathrm{C} .{ }^{1} \mathrm{H}$ NMR $\left(\mathrm{CDCl}_{3}\right) \delta: 3.40(\mathrm{~s}, 3 \mathrm{H}$, $\left.\mathrm{N}-\mathrm{CH}_{3}\right), 4.84(\mathrm{~s}, 2 \mathrm{H}, \mathrm{CH}), 7.12-7.80(\mathrm{~m}, 8 \mathrm{H}, \mathrm{Ar} H), 8.58(\mathrm{~s}, 1 \mathrm{H}, \mathrm{NCH})$. IR (KBr): 3522, 1636, 1428, 1342, $1165 \mathrm{~cm}^{-1}$; MS m/z: $347\left[\mathrm{M}^{+}\right], 349\left[\mathrm{M}^{+}+2\right]$; Anal. calcd for $\mathrm{C}_{16} \mathrm{H}_{14} \mathrm{~N}_{3} \mathrm{SO}_{2} \mathrm{Cl}$ : C, 55.25; H, 4.03; N, 12.09; Found: C, 55.23; $\mathrm{H}, 4.08 ; \mathrm{N}, 12.12$. Details of crystal data and structure refinement have been provided in Table 2 \& Fig. 4. Supplementary crystallographic data have been deposited with the CCDC number 804082 . These data can be obtained free of charge from Cambridge Crystallographic Data Centre via www.ccdc.cam. ac.uk/data request/cif.

$N$-(2-Methyl-benzylidene)- $N$ '-(1-methyl-2,2-dioxo-2,3-dihydro- $1 H$ $2 \lambda^{6}$-benzo[c] $[1,2]$ thiazin-4-ylidene)-hydrazine (6d):

Greenish yellow crystals; M.p. $148-150^{\circ} \mathrm{C} .{ }^{1} \mathrm{H}$ NMR $\left(\mathrm{CDCl}_{3}\right) \delta: 2.42(\mathrm{~s}$, $\left.3 \mathrm{H}, \mathrm{Ar}-\mathrm{CH}_{3}\right), 3.38\left(\mathrm{~s}, 3 \mathrm{H}, \mathrm{N}-\mathrm{CH}_{3}\right), 4.88\left(\mathrm{~s}, 2 \mathrm{H}, \mathrm{CH}_{2}\right), 7.12-8.02(\mathrm{~m}, 8 \mathrm{H}, \mathrm{Ar} H)$, 8.47 (s, 1H, NCH). IR (KBr): 3507, 1608, 1453, 1336, $1155 \mathrm{~cm}^{-1} ; \mathrm{MS} \mathrm{m} / z$ : 327 [ $\mathrm{M}^{+}$]; Anal. calcd for $\mathrm{C}_{17} \mathrm{H}_{17} \mathrm{~N}_{3} \mathrm{SO}_{2}: \mathrm{C}, 62.39 ; \mathrm{H}, 5.20 ; \mathrm{N}, 12.84$; Found: $\mathrm{C}$, 62.38; H, 5.21; N, 12.85

$\mathrm{N}$-(4-Methyl-benzylidene)- $\mathrm{N}$-(1-methyl-2,2-dioxo-2,3-dihydro- $1 \mathrm{H}$ $2 \lambda^{6}$-benzo $[c][1,2]$ thiazin-4-ylidene)-hydrazine (6e):

Yellow crystals; M.p. $189-190^{\circ} \mathrm{C} .{ }^{1} \mathrm{H}$ NMR $\left(\mathrm{CDCl}_{3}\right) \delta: 2.43$ (s, 3H, Ar$\left.\mathrm{CH}_{3}\right), 3.37\left(\mathrm{~s}, 3 \mathrm{H}, \mathrm{N}-\mathrm{CH}_{3}\right), 4.85\left(\mathrm{~s}, 2 \mathrm{H}, \mathrm{CH}_{2}\right), 7.10-7.74(\mathrm{~m}, 8 \mathrm{H}, \mathrm{ArH}), 8.57(\mathrm{~s}$, 
1H, NCH). IR (KBr): 3515, 1610, 1445, 1325, $1150 \mathrm{~cm}^{-1} ; \mathrm{MS} \mathrm{m} / z: 327\left[\mathrm{M}^{+}\right]$; Anal. calcd for $\mathrm{C}_{17} \mathrm{H}_{17} \mathrm{~N}_{3} \mathrm{SO}_{2}$ : C, 62.39; H, 5.20; N, 12.84; Found: $\mathrm{C}, 62.41 ; \mathrm{H}$, $5.24 ; \mathrm{N}, 12.81$.

$N$-(2-Nitro-benzylidene)- $N$-(1-methyl-2,2-dioxo-2,3-dihydro-1H-2 $\lambda^{6}$ benzo[c][1,2]thiazin-4-ylidene)-hydrazine (6f):

Yellow crystalline powder; M.p. $292-295^{\circ} \mathrm{C} .{ }^{1} \mathrm{H}$ NMR $\left(\mathrm{CDCl}_{3}\right) \delta: 3.38(\mathrm{~s}$, $\left.3 \mathrm{H}, \mathrm{N}-\mathrm{CH}_{3}\right), 4.86\left(\mathrm{~s}, 2 \mathrm{H}, \mathrm{CH}_{2}\right), 7.10-7.85(\mathrm{~m}, 8 \mathrm{H}, \mathrm{Ar} H), 8.53(\mathrm{~s}, 1 \mathrm{H}, \mathrm{NCH})$. IR (KBr): 3518, 1638, 1426, 1345, $1160 \mathrm{~cm}^{-1}$.MS m/z: 358.09 [M+]; Anal. calcd for $\mathrm{C}_{16} \mathrm{H}_{14} \mathrm{~N}_{4} \mathrm{SO}_{4}$ : C, 53.63; $\mathrm{H}, 3.91 ; \mathrm{N}, 15.64$; Found: $\mathrm{C}, 53.60$; $\mathrm{H}, 3.93$; $\mathrm{N}, 15.66$.

$N$-(6-Bromo-1-methyl-2,2-dioxo-2,3-dihydro- $1 H-2 \lambda^{6}$-benzo $[c][1,2]$ thiazin-4-ylidene)- $N$-(3-chloro-benzylidene)-hydrazine (6g):

Yellow powder, M.p. $197-198^{\circ} \mathrm{C} .{ }^{1} \mathrm{H}$ NMR $\left(\mathrm{CDCl}_{3}\right) \delta$ : 3.38 (s, 3H, $\left.\mathrm{N}-\mathrm{CH}_{3}\right), 4.84$ (s, 2H, $\left.\mathrm{CH}_{2}\right), 6.99-7.87$ (m, 7H, $\left.\mathrm{ArH}\right), 8.58$ (s, 1H, NCH). IR (KBr): $3518,1636,1416,1333,1146 \mathrm{~cm}^{-1} ; \mathrm{MS} m / z: 425\left[\mathrm{M}^{+}\right], 427\left[\mathrm{M}^{+}+2\right], 429$ $\left[\mathrm{M}^{+}+4\right]$; Anal. calcd for $\mathrm{C}_{16} \mathrm{H}_{13} \mathrm{~N}_{3} \mathrm{SO}_{2} \mathrm{BrCl}$ : $\mathrm{C}, 45.02 ; \mathrm{H}, 3.05$; N, 9.85; Found: C, 45.00; H, 3.04; N, 9.87.

$N$-(6-Bromo-1-methyl-2,2-dioxo-2,3-dihydro- $1 H$-2 $2 \lambda^{6}$-benzo $[c][1,2]$ thiazin-4-ylidene)- $N$-(4-chloro-benzylidene)-hydrazine (6h):

Yellow crystalline powder; M.p. $210-211^{\circ} \mathrm{C} .{ }^{1} \mathrm{H}$ NMR $\left(\mathrm{CDCl}_{3}\right) \delta: 3.38(\mathrm{~s}$, $\left.3 \mathrm{H}, \mathrm{N}-\mathrm{CH}_{3}\right), 4.84\left(\mathrm{~s}, 2 \mathrm{H}, \mathrm{CH}_{2}\right), 6.99-7.80(\mathrm{~m}, 7 \mathrm{H}, \mathrm{Ar} H), 8.59$ (s, $\left.1 \mathrm{H}, \mathrm{NCH}\right)$. IR (KBr): $3510,1625,1426,1340,1150 \mathrm{~cm}^{-1}$; MS m/z: $425\left[\mathrm{M}^{+}\right], 427\left[\mathrm{M}^{+}+2\right], 429$ $\left[\mathrm{M}^{+}+4\right]$. Anal. calcd for $\mathrm{C}_{16} \mathrm{H}_{13} \mathrm{~N}_{3} \mathrm{SO}_{2} \mathrm{BrCl}$ : C, 45.02; H, 3.05; N, 9.85; Found: C, 44.99; H, 3.03; N, 9.83.

$N$-(6-Bromo-1-methyl-2,2-dioxo-2,3-dihydro- $1 H$-2 $\lambda^{6}$-benzo $[c][1,2]$ thiazin-4-ylidene)- $\boldsymbol{N}$-(4-fluoro-benzylidene)-hydrazine (6i):

Yellow crystalline powder; M.p. 196-198 ${ }^{\circ} \mathrm{C} .{ }^{1} \mathrm{H} \mathrm{NMR}\left(\mathrm{CDCl}_{3}\right)$ ) : 3.37 (s, $\left.3 \mathrm{H}, \mathrm{N}-\mathrm{C} H_{3}\right), 4.84\left(\mathrm{~s}, 2 \mathrm{H}, \mathrm{CH}_{2}\right), 6.98-7.86(\mathrm{~m}, 7 \mathrm{H}, \mathrm{Ar} H), 8.58(\mathrm{~s}, 1 \mathrm{H}, \mathrm{NCH})$. IR (KBr): 3530, 1618, 1412, 1340, $1153 \mathrm{~cm}^{-1}$; MS m/z: 409 [M+1, $411\left[\mathrm{M}^{+}+2\right]$; Anal. calcd for $\mathrm{C}_{16} \mathrm{H}_{13} \mathrm{~N}_{3} \mathrm{SO}_{2} \mathrm{BrF}$ : C, 46.83; H, 3.17; N, 10.24; Found: C, 46.80; $\mathrm{H}, 3.21 ; \mathrm{N}, 10.27$.

$N$-(6-Bromo-1-methyl-2,2-dioxo-2,3-dihydro-1 $H$-2 $\lambda^{6}$-benzo $[c][1,2]$ thiazin-4-ylidene)- $N$-(4-methyl-benzylidene)-hydrazine (6j):

Yellow needle like crystals; M.p. $184-185^{\circ} \mathrm{C} .{ }^{1} \mathrm{H}$ NMR $\left(\mathrm{CDCl}_{3}\right) \delta: 2.43$ (s, $\left.3 \mathrm{H}, \mathrm{Ar}-\mathrm{CH}_{3}\right), 3.37$ (s, 3H, N-CH$), 4.86\left(\mathrm{~s}, 2 \mathrm{H}, \mathrm{CH}_{2}\right), 6.98-7.75(\mathrm{~m}, 7 \mathrm{H}, \mathrm{Ar} H)$, 8.59 (s, $1 \mathrm{H}, \mathrm{NCH}$ ). IR (KBr): $3515,1640,1417,1336,1155 \mathrm{~cm}^{-1}$; MS m/z: 405
$\left[\mathrm{M}^{+}\right], 407\left[\mathrm{M}^{+}+2\right]$; Anal. calcd for $\mathrm{C}_{17} \mathrm{H}_{16} \mathrm{~N}_{3} \mathrm{SO}_{2} \mathrm{Br}$ : C, 50.25; H, 3.94; N, 10.34; Found: C, 50.28; H, 3.95; N, 10.31

$N$-(6-Bromo-1-methyl-2,2-dioxo-2,3-dihydro- $1 H$-2 $\lambda^{6}$-benzo $[c][1,2]$ thiazin-4-ylidene)- $N$ '-(4-nitro-benzylidene)-hydrazine (6k):

Yellow needle like crystals; M.p. $244-246^{\circ} \mathrm{C} .{ }^{1} \mathrm{H}$ NMR $\left(\mathrm{CDCl}_{3}\right) \delta: 3.40$ (s, $\left.3 \mathrm{H}, \mathrm{N}-\mathrm{CH}_{3}\right), 4.87\left(\mathrm{~s}, 2 \mathrm{H}, \mathrm{CH}_{2}\right), 7.10-7.82(\mathrm{~m}, 7 \mathrm{H}, \mathrm{Ar} H), 8.56(\mathrm{~s}, 1 \mathrm{H}, \mathrm{NCH})$ IR (KBr): 3509, 1642, 1413, 1315, $1145 \mathrm{~cm}^{-1}$; MS m/z: $436\left[\mathrm{M}^{+}\right], 438\left[\mathrm{M}^{+}+2\right]$; Anal. calcd for $\mathrm{C}_{16} \mathrm{H}_{13} \mathrm{~N}_{4} \mathrm{SO}_{4} \mathrm{Br}$ : C, 43.94; H, 2.97; N, 12.81; Found: C, 43.92; $\mathrm{H}, 2.98 ; \mathrm{N}, 12.84$.

$N$-Anthracen-9-yl-methylene- $N$-(6-Bromo-1-methyl-2,2-dioxo-2,3dihydro-1H-2 $\lambda^{6}$-benzo[c][1,2] thiazin-4-ylidene)-hydrazine (61):

Dark orange thin crystals; M.p. $215-218^{\circ} \mathrm{C} .{ }^{1} \mathrm{H}$ NMR $\left(\mathrm{CDCl}_{3}\right) \delta: 3.44$ (s, $\left.3 \mathrm{H}, \mathrm{N}-\mathrm{CH}_{3}\right), 4.85\left(\mathrm{~s}, 2 \mathrm{H}, \mathrm{CH}_{2}\right), 7.05-8.25(\mathrm{~m}, 12 \mathrm{H}, \mathrm{Ar} H), 8.52(\mathrm{~s}, 1 \mathrm{H}, \mathrm{NCH})$. IR (KBr): 3528, 1621, 1415, 1336, $1152 \mathrm{~cm}^{-1} ; \mathrm{MS} \mathrm{m} / z: 491\left[\mathrm{M}^{+}\right], 493\left[\mathrm{M}^{+}+2\right]$; Anal. calcd for $\mathrm{C}_{24} \mathrm{H}_{18} \mathrm{~N}_{3} \mathrm{SO}_{2} \mathrm{Br}$ : C, 58.54; H, 3.66; N, 8.54; Found: C, 58.51; $\mathrm{H}, 3.64 ; \mathrm{N}, 8.57$.

4-[(6-Bromo-1-methyl-2,2-dioxo-2,3-dihydro- $1 H$-2 $\lambda^{6}$-benzo $[c][1,2]$ thiazin-4-ylidene)-hydrazonomethyl]-phenol (6m):

Yellow powder; M.p. $209-210^{\circ} \mathrm{C} .{ }^{1} \mathrm{H}$ NMR $\left(\mathrm{CDCl}_{3}\right) \delta: 3.41$ (s, 3H, $\mathrm{NCH}_{3}$ ), $4.94\left(\mathrm{~s}, 2 \mathrm{H}, \mathrm{CH}_{2}\right), 7.03-8.08(\mathrm{~m}, 7 \mathrm{H}, \mathrm{Ar} H), 8.62(\mathrm{~s}, 1 \mathrm{H}, \mathrm{NCH}), 9.98(\mathrm{~s}, 1 \mathrm{H}$, ArOH). IR (KBr): 3495, 1629, 1438, 1332, $1145 \mathrm{~cm}^{-1} ; \mathrm{MS} \mathrm{m} / z$ : $407\left[\mathrm{M}^{+}\right], 409$ $\left[\mathrm{M}^{+}+2\right]$; Anal. calcd for $\mathrm{C}_{17} \mathrm{H}_{16} \mathrm{~N}_{3} \mathrm{SO}_{3} \mathrm{Br}$ : C, 47.05; H, 3.43; N, 10.29; Found: C, $47.04 ; \mathrm{H}, 3.45 ; \mathrm{N}, 10.31$.

$N$-(6-Bromo-1-methyl-2,2-dioxo-2,3-dihydro- $1 H$-2 $\lambda^{6}$-benzo $[c][1,2]$ thiazin-4-ylidene)- $N$ '-(3,4-dimethoxy-benzylidene)-hydrazine (6n):

Light orange crystalline powder; M.p. $225-227^{\circ} \mathrm{C} .{ }^{1} \mathrm{H}$ NMR $\left(\mathrm{CDCl}_{3}\right) \delta$ : $3.08\left(\mathrm{~s}, 6 \mathrm{H}, \mathrm{Ar}-\mathrm{OCH}_{3}\right), 3.36\left(\mathrm{~s}, 3 \mathrm{H}, \mathrm{N}-\mathrm{CH}_{3}\right), 4.89\left(\mathrm{~s}, 2 \mathrm{H}, \mathrm{CH}_{2}\right), 6.72-7.73(\mathrm{~m}$, $6 \mathrm{H}, \mathrm{ArH}), 8.56(\mathrm{~s}, 1 \mathrm{H}, \mathrm{NCH})$. IR (KBr): $3505,1615,1401,1355,1145 \mathrm{~cm}^{-1}$; MS m/z: $451\left[\mathrm{M}^{+}\right], 453\left[\mathrm{M}^{+}+2\right]$; Anal. calcd for $\mathrm{C}_{18} \mathrm{H}_{18} \mathrm{~N}_{3} \mathrm{SO}_{4} \mathrm{Br}$ : C, $47.79 ; \mathrm{H}$, 3.98; N, 9.29; Found: C, 47.82; H, 4.00; N, 9.32.

4-[(6-Bromo-1-methyl-2,2-dioxo-2,3-dihydro- $1 H$-2 $\lambda^{6}$-benzo $[c][1,2]$ thiazin-4-ylidene)-hydrazonomethyl]-2-methoxy-phenol (6o):

Yellow crystalline solid; M.p. $252-253^{\circ} \mathrm{C} .{ }^{1} \mathrm{H}$ NMR $(\mathrm{CDCl})$ ) $\delta: 3.10(\mathrm{~s}, 3 \mathrm{H}$ Ar- $\left.\mathrm{OCH}_{3}\right), 3.32\left(\mathrm{~s}, 3 \mathrm{H}, \mathrm{N}-\mathrm{CH}_{3}\right), 4.90\left(\mathrm{~s}, 2 \mathrm{H}, \mathrm{CH}_{2}\right), 6.96-8.02(\mathrm{~m}, 6 \mathrm{H}, \mathrm{ArH})$ $8.58(\mathrm{~s}, 1 \mathrm{H}, \mathrm{NCH}), 9.56(\mathrm{~s}, 1 \mathrm{H}, \mathrm{OH})$. IR $(\mathrm{KBr}): 3503,1615,1410,1350,1148$ $\mathrm{cm}^{-1}$; MS m/z: $437\left[\mathrm{M}^{+}\right], 439\left[\mathrm{M}^{+}+2\right]$; Anal. calcd for $\mathrm{C}_{17} \mathrm{H}_{16} \mathrm{~N}_{3} \mathrm{SO}_{4} \mathrm{Br}: \mathrm{C}, 46.58$; H, 3.65; N, 9.59; Found: C, 46.55; H, 3.67; N, 9.57.

Table 1: Reaction parameters and yields for the synthesis of 6a-0.

\begin{tabular}{|c|c|c|c|c|c|c|}
\hline Entry & Reactant & Product & Thermal Reaction Conditions & Yield (\%) & $\begin{array}{c}\text { Ultrasonic } \\
\text { Reaction conditions }\end{array}$ & Yield (\%) \\
\hline 1 & $5 \mathrm{a}$ & $6 \mathrm{a}$ & Reflux:60 minutes & 76.0 & $35^{\circ} \mathrm{C} ; 2.0$ minutes & 94.4 \\
\hline 2 & $5 \mathrm{a}$ & $6 \mathrm{~b}$ & Reflux:60 minutes & 77.5 & $30^{\circ} \mathrm{C} ; 2.5$ minutes & 92.6 \\
\hline 3 & $5 \mathrm{a}$ & $6 \mathrm{c}$ & Reflux:60 minutes & 75.7 & $30^{\circ} \mathrm{C} ; 2.0$ minutes & 93.2 \\
\hline 4 & $5 \mathrm{a}$ & $6 \mathrm{~d}$ & Reflux:125 minutes & 80.5 & $30^{\circ} \mathrm{C} ; 2.0$ minutes & 95.2 \\
\hline 5 & $5 \mathrm{a}$ & $6 \mathrm{e}$ & Reflux:90 minutes & 79.1 & $35^{\circ} \mathrm{C} ; 2.5$ minutes & 94.8 \\
\hline 6 & $5 \mathrm{a}$ & $6 \mathrm{f}$ & Reflux:35 minutes & 75.8 & $30^{\circ} \mathrm{C} ; 2.5$ minutes & 90.8 \\
\hline 7 & $5 \mathrm{~b}$ & $6 \mathrm{~g}$ & Reflux:60 minutes & 76.3 & $35^{\circ} \mathrm{C} ; 3.0$ minutes & 91.8 \\
\hline 8 & $5 \mathrm{~b}$ & $6 \mathrm{~h}$ & Reflux:60 minutes & 76.6 & $35^{\circ} \mathrm{C} ; 2.5$ minutes & 92.6 \\
\hline 9 & $5 \mathrm{~b}$ & $6 \mathrm{i}$ & Reflux:45 minutes & 78.8 & $40^{\circ} \mathrm{C} ; 3.0$ minutes & 93.0 \\
\hline 10 & $5 \mathrm{~b}$ & $6 \mathrm{j}$ & Reflux:100 minutes & 8.0 minutes & 93.0 \\
\hline 11 & $5 \mathrm{~b}$ & $6 \mathrm{k}$ & Reflux: 110 minutes & 84.1 & $35^{\circ} \mathrm{C} ; 3.0$ minutes & 91.2 \\
\hline 12 & $5 \mathrm{~b}$ & 61 & Reflux:80 minutes & 80.4 & $35^{\circ} \mathrm{C} ; 3.0$ minutes & 99.6 \\
\hline 13 & $5 \mathrm{~b}$ & $6 \mathrm{~m}$ & Reflux:90 minutes & 79.2 & $35^{\circ} \mathrm{C} ; 2.5$ minutes & 95.4 \\
\hline 14 & $5 \mathrm{~b}$ & $6 \mathrm{n}$ & Reflux:70 minutes & 84.1 & $40^{\circ} \mathrm{C} ; 3.0$ minutes & 92.6 \\
\hline 15 & $5 \mathrm{~b}$ & $6 \mathrm{o}$ & Reflux: 40 minutes & 82.9 & $40^{\circ} \mathrm{C} ; 2.5$ minutes & 93.2 \\
\hline
\end{tabular}

X-RAY CRYSTALLOGRAPHY

X-ray data were collected at $296 \mathrm{~K}$ on a Bruker KAPPA APEX II diffractometer using Mo K $\alpha$ X-ray $(0.71073 \AA)$ source and a graphite monochromator. The unit cell dimensions were obtained from least-squares fit to setting angles of about 25 reflections. Multi-scan absorption corrections were applied. SAINT ${ }^{20}$ was used for the cell refinement and data reduction, while SHELXS- $97^{21}$ was used for structure solution and refinement. ORTEP-3 for Windows ${ }^{22}$, PLATON ${ }^{23}$ and WinGX ${ }^{24}$ were used for molecular graphics. In the refinement procedure, all the non-hydrogen atoms were refined with anisotropic displacement parameters. 
The aromatic and aliphatic hydrogen atoms were positioned geometrically and treated as riding atom over their parent carbon atoms. Details of crystal data and structure refinement have been provided in Table 2 \& Fig. 4. Supplementary crystallographic data have been deposited with the CCDC number 804082. These data can be obtained free of charge from Cambridge Crystallographic Data Centre via www.ccdc.cam.ac.uk/data request/cif.

ANTI-BACTERIAL ASSAY:

Anti-bacterial assay of the synthesized compounds (dissolved in dimethylformamide) was carried out by Agar Well Diffusion method ${ }^{25}$ against the human pathogenic bacteria. Suspensions of five strains of bacteria i.e., Escherichia coli, Salmonella typhimurium, Pseudomonas aeruginosa, Staphylococcus aureus, and Salmonella typhimurium were adjusted with sterile saline to a concentration of $1.0 \times 10^{5}$ colony forming unit $(\mathrm{CFU}) / \mathrm{ml}$. The inocula were prepared daily and stored at $+4^{\circ} \mathrm{C}$ until use. Dilutions of the inocula were cultured on solid medium to verify the absence of contamination and to check the validity of the inoculum. The compounds under investigation were applied to the wells of $6 \mathrm{~mm}$ diameter at $1 \mathrm{mg}$ per $\mathrm{ml}$ of DMF in addition to 0 (control) and the standards, ampicillin \& ciprofloxacin. The plates were incubated at $37^{\circ} \mathrm{C}$ for 24 hours and zone of inhibition was measured as an average of three values.

\section{RESULTS AND DISCUSSION}

1-Methyl-2,2-dioxo-2,3-dihydro-1 $H-2 \lambda^{6}$-benzo[c][1,2]thiazin-4-one (4a) and 6-bromo-1-methyl-2,2-dioxo-2,3-dihydro-1 $H$-2 $\lambda^{6}$-benzo[c][1,2] thiazin-4one (4b) were synthesized by condensation of methane sulfonyl chloride with methyl anthranilate and 5-bromo methyl anthranilate respectively followed by $\mathrm{N}$-methylation using methyl iodide. Sulfonamide formation between methyl anthranilate and methane sulfonyl chloride was carried out under the influence of ultrasonic waves and the reaction was completed in far less time (40 minutes) than synthesized by literature procedure in diethyl ether (3.5 hours). However, synthesis of bromo derivative (4b) took relatively longer time (3.5 hours) even at elevated temperature $\left(60-70^{\circ} \mathrm{C}\right)$. Poor reactivity of 5-bromo methyl anthranilate with methane sulfonyl chloride may be attributed to the presence of bromine on the ring making amino group relatively weaker nucleophile. Similar behavior was shown by the un/ bromo substituted intermediates (2a-b) in subsequent $\mathrm{N}$-alkylation. However, cyclization of ring had no such effect, which may be due to generation of carbanion at methyl group of the sulfonamide. Its attack on to the electrophilic carbon of the carbonyl group is independent of the presence or absence of an electron withdrawing group on the benzene ring. In the next step, 1-methyl-2,2-dioxo-2,3-dihydro- $1 H$-2 $2 \lambda^{6}$-benzo $[c][1,2]$ thiazin4-ones (4a) and 6-bromo-1-methyl-2,2-dioxo-2,3-dihydro-1H-2 $\lambda^{6}$-benzo[c] $[1,2]$ thiazin-4-one (4b) were reacted with hydrazine hydrate to get 1-methyl2,2-dioxo-2,3-dihydro-1 $H$-2 $\lambda^{6}$-benzo[c] [1,2]thiazin-4-ylidene)-hydrazine (5a) and 6-bromo 1-methyl-2,2-dioxo-2,3-dihydro- $1 H$-2 $2 \lambda^{6}$-benzo[ $\left.c\right][1,2]$ thiazin-4ylidene)-hydrazine (5b) respectively. These were further reacted with different aldehydes in ultrasonic bath to get the title compounds (6a-o) in excellent yields (See Table 1).

It was observed that using ultrasonic waves, reaction times have been considerably reduced with improved yields (for comparison of yields, reaction conditions and reaction times, see Table 1). All of the synthesized compounds are characterized through spectroscopic techniques. In order to determine the stereochemistry ( $E$ or $Z$ configuration), (as it could not be revealed through ${ }^{1} \mathrm{H}$ NMR spectroscopy) a single crystal of $N$-(4-chloro-benzylidene)- $N$ '-(1-methyl2,2-dioxo-2,3-dihydro-1 $H$-2 $\lambda^{6}$-benzo[c][1,2]thiazin-4-ylidene)-hydrazine (6c) was grown in methanol and studied by X-ray crystallography. In the crystal structure of the subjected molecule, $\mathrm{C}=\mathrm{N}$ linkage adopts an $E$ configuration and the non-planer thiazine ring $(\mathrm{C} 1 / \mathrm{C} 6 / \mathrm{C} 7 / \mathrm{C} 8 / \mathrm{N} 1 / \mathrm{S} 1)$ with the r.m.s deviation of $0.2334 \AA$ assumes the envelop shape. The two aromatic rings i.e. fused benzene and chlorophenyl rings form the dihedral angles of $10.19(0.24)^{\circ}$ and $10.56(0.24)^{\circ}$ respectively with respect to the thiazine ring. The structure is stabilized by the weak hydrogen bonding $(\mathrm{C}---\mathrm{H} . . . \mathrm{O})$ interactions. The crystal structure indicates $E$ configuration of $\mathrm{C}=\mathrm{N}$ bond for this specific compound. It is assumed that rest of the compounds too have same $(E)$ configuration which is in consistent with literature studies ${ }^{14}$.

Table 2: Crystal data and structure refinement for compound 6c

\begin{tabular}{|c|c|c|c|}
\hline Structural formula & $\mathrm{C}_{16} \mathrm{H}_{14} \mathrm{~N}_{3} \mathrm{O}_{2} \mathrm{SCl}$ & Cell Volume & $1577.52(26) \AA^{3}$ \\
\hline Formula weight & 347.80 & $\mathrm{Z}$ & 4 \\
\hline Crystal system & Monoclinic & Calculated density $/\left(\mathrm{g} \cdot \mathrm{cm}^{-3}\right)$ & 1.46 \\
\hline Space group & $\mathrm{P} 121 / \mathrm{n} 1$ & Absorption coefficient/ $\mathrm{mm}^{-1}$ & 0.3877 \\
\hline $\mathrm{T}(\mathrm{K})$ & $296(2) \mathrm{K}$ & Crystal size $\left(\mathrm{mm}^{3}\right)$ & $0.29 \times 0.17 \times 0.19$ \\
\hline$A(\AA)$ & $8.3157(27)$ & Reflection collected/unique & $16179 / 3955$ \\
\hline$B(\AA)$ & $12.8986(39)$ & Range for data collection $\left(^{\circ}\right)$ & $2.1-28.5$ \\
\hline$C(\AA)$ & $14.7873(42)$ & Goodness-of-fit on $F^{2}$ & 0.876 \\
\hline$\Delta\left(^{\circ}\right)$ & 90.00 & $F(000)$ & 719.9 \\
\hline$\Delta\left(^{\circ}\right)$ & $95.962(15)$ & Absorption correction & Multi-Scan \\
\hline$\Delta\left(^{\circ}\right)$ & 90.00 & Refinement method & Full-matrix least squares on $F^{2}$ \\
\hline
\end{tabular}

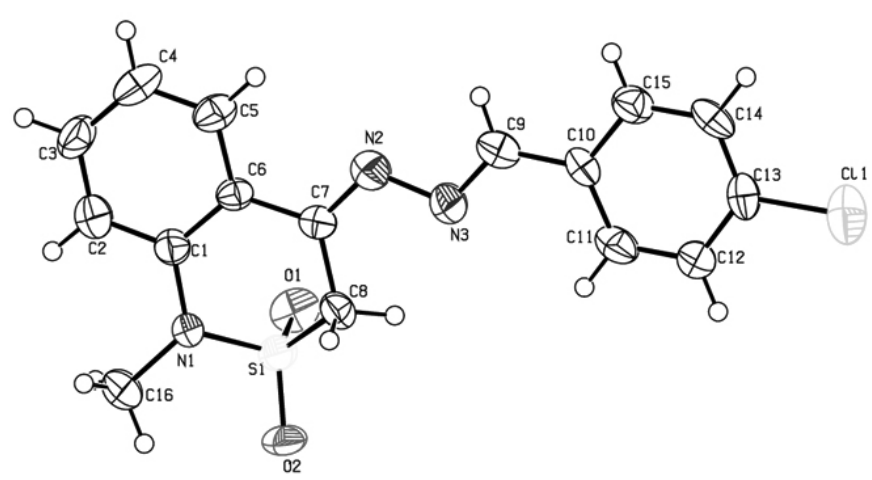

Figure 4: ORTEP diagram for $\mathbf{6 c}$ with the $50 \%$ probability level of the thermal ellipsoids
Anti-bacterial activities of the compounds under investigation are presented in Table 3 in comparison with reference drugs Ampicillin and Ciprofloxacin. assay revealed that most of the derivatives are active against pseudomonas aeruginosa and some are active against Salmonella typhimurium; however, $\mathbf{6} \mathbf{i}$ was found the only active compound against $E$. coli. Moreover, the results also showed that amongst these synthesized compounds $\mathbf{6 a}, \mathbf{6} \mathbf{6}, \mathbf{6 h}, \mathbf{6 i}$ and $\mathbf{6 j}$ has shown good activities. From the results, it is evident that Pseudomonas aeruginosa is the most sensitive bacterial species for these compounds, while E. coli and Enterococcus faecalis were found as the most resistant species. These compounds are mainly active against Gram negative bacteria such as Pseudomonas aeruginosa and Salmonella typhimurium while they have shown negligible activities against Gram positive bacteria. 
Table 3: Anti-bacterial Activity of Tested Compounds (Zone of inhibition in mm).

\begin{tabular}{|c|c|c|c|c|c|}
\hline Compound & $\begin{array}{c}\text { Pseudomonas } \\
\text { aeruginosa }\end{array}$ & Escherichia coli & $\begin{array}{c}\text { Salmonella } \\
\text { typhimurium }\end{array}$ & $\begin{array}{c}\text { Enterococcus } \\
\text { faecalis }\end{array}$ & $\begin{array}{c}\text { Staphylococcus } \\
\text { aureus }\end{array}$ \\
\hline 6a & 8.55 & - & - & - & 12.53 \\
\hline $6 \mathrm{~b}$ & 10.10 & - & 11.12 & - & - \\
\hline 6c & - & - & 9.87 & - & - \\
\hline 6d & - & - & 8.89 & - & - \\
\hline $6 \mathrm{e}$ & 9.33 & - & - & - & - \\
\hline $6 \mathrm{f}$ & 9.30 & - & - & - & 12.84 \\
\hline $6 \mathrm{~g}$ & - & - & - & - & - \\
\hline $6 \mathrm{~h}$ & 11.47 & - & 9.84 & - & - \\
\hline $6 \mathrm{i}$ & 10.27 & 9.61 & - & - & - \\
\hline $6 \mathrm{j}$ & - & - & 9.98 & 9.98 & - \\
\hline 61 & 8.95 & - & - & - & - \\
\hline 6m & 9.15 & - & 8.09 & - & - \\
\hline 6n & 9.30 & - & - & - & 30.95 \\
\hline Ampicillin & - & 19.47 & 25.06 & 30.54 & 20.54 \\
\hline Ciprofloxacin & 23.93 & 31.40 & 25.0 & 21.6 & \\
\hline
\end{tabular}

\section{CONCLUSION}

Synthesis of $\quad N$-benzylidene- $N$ '-(1-methyl-2,2-dioxo-2,3-dihydro- $1 H$ $2 \lambda^{6}$-benzo $[c][1,2]$ thiazin-4-ylidene)-hydrazines and their evaluation for antibacterial potential is reported. Some of the synthesized compounds have shown good anti-bacterial activity and this study may be useful to synthesize more biologically active compounds through further derivatization.

\section{ACKNOWLEDGEMENTS}

Muhammad Shafiq gratefully acknowledges Higher Education Commision, Islamabad Pakistan for research scholarship under its indigenous $\mathrm{PhD}$ Scholarship 5000 Scheme. Assistance of M/s Qarshi Research International, Hattar, Pakistan to M. Shafiq is also greatly acknowledged for the provision of facilities of anti-bacterial assay.

\section{REFERENCES}

1. P. R. Hanson, D. A. Probst, R. E. Robinson, M. Yau, Tetrahedron Lett. 40, 4761 (1999).

2. K. H. Ahn, C. Ham, S-K. Kim, C-W. Cho, J. Org. Chem. 62, 7047 (1997).

3. W. Oppolzer, A. J. Kingma, S. K. Pillai, Tetrahedron Lett. 32, 4893 (1991).

4. F. Flugel, D. Bente, T. Itil, Dtsch. Med. Wochenschr. 85, 2199 (1960).

5. S. Hayashi, H. Ueki, Y. Sako, T. Ashunura, K. Hayashi, K.Takase, Kumamoto Pharm. Bull. 5, 51, (1962).

6. J.V. Braun, Chem. Ber. 56, 2332 (1923).

7. M. Harmata, N.L. Calkins, R.G. Baughman, C. L. Barnes, J. Org. Chem. 71, 3650 (2006)
8. Y. Misu, H.Togo, Org. Biomol. Chem. 1, 1342-1346 (2003).

9. S. Rollas, S. G. Küçükgüzel, Molecules, 12, 1910-1939 (2007).

10. C. Loncle, J. M. Brunel, N. Vidal, M. Dherbomez, Y. Letourneux, Eur. J. Med. Chem. 39, 1067-1071 (2004).

11. S. N. Khattab, Molecules, 10, 1218-1228 (2005).

12. M. Zia-ur-Rehman, J. A. Choudary, S. Ahmad, Bull. Korean Chem. Soc. 26, 1771 (2005).

13. M. Zia-ur-Rehman, J. A. Choudary, S. Ahmad, H. L. Siddiqui, Chem. Pharm. Bull. (Japan), 54, 1175 (2006).

14. M. Ahmad, H. L. Siddiqui, M. Zia-ur-Rehman, M. Parvez, Eur. J. Med. Chem. 45, 698 (2010).

15. M. Shafiq, M. N. Tahir, I. U. Khan, M. N. Arshad, M. H. Khan, Acta Cryst. $E, \mathbf{6 5}, 0955$ (2009).

16. M. N. Tahir, M. Shafiq, I. U. Khan, W. A. Siddiqui, M. N. Arshad, Acta Cryst. E, 64, o557 (2008).

17. M. Shafiq, M. N. Tahir, I. U. Khan, M. N. Arshad, M. N. Asghar, Acta Cryst. E, 65, o1182 (2009).

18. J. G. Lombardino, J. Heterocyclic Chem. 315-317 (1971).

19. B. Loev, K. M. Sander, J. Heterocyclic Chem., 4, 403 (1967).

20. Bruker SADABS and Bruker AXS Inc., Madison, Wisconsin, USA (2007).

21. G. M. Sheldrick, Acta Cryst. A, 64, 112-122 (2008).

22. L. J. Farrugia, J. Appl. Cryst. 30, 565 (1997).

23. L. Spek, Acta Cryst. D, 65, 148-155 (2009).

24. L. J. Farrugia, J. Appl. Cryst. 30, 565 (1999).

25. J. G. Colle, J. P. Duguid, A. G. Fraser, B. P. Marmion, "Practical Medical Biology", $13^{\text {th }}$ ed. By T. J. Mackie, J.E. McCartney, Churchill Livingstone, London, UK, 1989. 